\title{
COMPUTATION, DIMENSIONALITY, AND ZERO DISSIPATION LIMIT OF THE GINZBURG-LANDAU WAVE EQUATION
}

\author{
By \\ Yisong Yang
}

IMA Preprint Series \# 504

February 1989 


\title{
Computation, Dimensionality, and Zero Dissipation Limit of the Ginzburg-Landau Wave Equation
}

\author{
YISONG YANG $\dagger$
}

\begin{abstract}
In this paper we prove the convergence and obtain optimal error estimates of the Galerkin approximation scheme for computing spatially-periodic solutions of the Ginzburg-Landau wave equation. Using this convergent scheme, we carry out a series of numerical experiments towards an understanding of the finite dimensionality of the wave motions evolving from a small neighbourhood of the zero solution. In the zero dissipation limit, we show that the flow approach the solutions of the cubic Schrödinger equation.
\end{abstract}

AMS(MOS) subject classifications. 35Q20, 65M60, 76E30

1. Introduction. The evolution of the slowly varying envelope or amplitude of instability waves close to criticality in a wide range of problems, such as Poiseuille flow [16], Rayleigh-Bénard convection [11], and Taylor-Couette flow [6] in fluid dynamics, and turbulent motions of reactant concentrations in oscillatory chemical reactions [7], is governed by the complex Ginzburg-Landau wave equation. After a suitable rescaling, this equation can be written $[12,15]$ :

$$
i \psi_{t}+\left(1-i c_{0}\right) \psi_{x x}=i\left(c_{0} / c_{1}\right) \psi-\left(1+i c_{0} / c_{1}\right)|\psi|^{2} \psi
$$

where $t$ is the time variable, $x$ is the one-dimensional spatial coordinate, $\psi(x, t)$ represents the complex envelope or amplitude under discussion, $c_{0}$ and $c_{1}$ are real parameters, and $i=\sqrt{-1}$. It is known that Eq.(1.1) admits a family of plane-wave solutions of the form $\psi=C e^{i(\omega t+k x)}$ as well as some exact solutions of solitary wave, hole, and shock-type found in [13]. In general, the initial value problem of Eq.(1.1) cannot be solved analytically, and consequently, various numerical integration methods have to be used. In simulating the long-time behaviour of instability wave motions, spatially-periodic solutions have widely been adopted and a fairly good understanding of Eq.(1.1) has been reached. Numerically, Eq.(1.1) has been integrated by many authors $[9,10,4]$ by using the pseudospectral method $[5,2]$ and in the parameter region $c_{0}>0, c_{1}>0$ the route to chaos observed. Moreover, the convergence of this scheme has recently been justified [19]. Analytically, it is known that the initial value problem of Eq.(1.1) can be solved globally in the parameter regions $c_{0}>0, c_{1}>0$ and $c_{0}>0, c_{1}<0$ (in the latter case, the initial periodic state has to be small to ensure global existence of a solution), while, in the region $c_{0}<0, c_{1}>0$, blowup motions may evolve from arbitrarily small initial data [18], and that, in the parameter region $c_{0}>0, c_{1}>0$, all spatially-periodic solutions are trapped into a universal attractor

$\dagger$ Department of Mathematics and Statistics, University of Massachusetts and Institute for Mathematics and its Applications, University of Minnesota 
of finite fractal dimensions as $t \rightarrow \infty$ and the upper and lower bounds of the dimensions can be estimated in terms of the parameters $c_{0}>0, c_{1}>0$, and the basic periods [3,1].

In this paper, we first obtain the convergence and optimal error estimates of Galerkin's (finite element) method for computing spatially-periodic solutions of the Ginzburg-Landau wave equation (1.1) in the parameter regions $c_{0}>0, c_{1}>0$ and $c_{0}>0, c_{1}<0$. One of the obvious advantages of this method is that the finite dimensionality of the wave motions may more clearly be exhibited. Then, we use this convergent scheme to make a series of computations towards understanding the solutions evolving from a small neighbourhood of the zero solution (or motionless state) $\psi=0$. It is interesting to observe that the GinzburgLandau equation (1.1) behaves like a wave filter so that only low frequency modes can survive after a long-time period and high modes are all exhausted. This shows the wave motions are indeed of a finite dimensional character even in a finite time development. More importantly, in all of our examples, we see that a sideband analysis already provides an accurate prediction of the number of dimensions of the solutions. In Eq.(1.1), setting $c_{0}$ $=0$, we obtain the very well understood integrable cubic Schrödinger equation [20] which has no attractor. Hence it looks interesting to know what will happen to the solutions of the Ginzburg-Landau equation as $c_{0} \rightarrow 0^{+}$. Naturally, one expects that the solutions will tend to that of the cubic Schrödinger equations (in a suitable sense). Studies of this type may be found in $[8,17]$ for the zero dispersion limit of the Korteweg-de Vries equation. In our case, $c_{0} \rightarrow 0^{+}$is the zero dissipation limit. Due to the properties of the GinzburgLandau, and Schrödinger, equation, we can reach a strong convergence result: as $c_{0} \rightarrow 0^{+}$, the solutions of the Ginzburg-Landau equation approach the solution of the Schrödinger equation pointwise on any finite time interval.

2. Galerkin's approximation. The (spatially-periodic) initial value problem of the Ginzburg-Landau equation can be written:

$$
\left\{\begin{array}{l}
\psi_{t}=\left(i+c_{0}\right) \psi_{x x}+\left(c_{0} / c_{1}\right) \psi+\left(i-c_{0} / c_{1}\right)|\psi|^{2} \psi,-\infty<x<\infty, t>0 \\
\psi(x, 0)=\psi^{0}(x), \quad \psi^{0}(x)=\psi^{0}(x+l), \quad-\infty<x<\infty
\end{array}\right.
$$

where $l>0$ is the basic period.

Let $(\cdot, \cdot)_{2}$ denote the usual inner product of the complex-valued function space $L^{2}(0, l)$. Then $L^{2}(0, l)$ has a standard orthonormal basis

$$
\left\{e_{n}(x)\right\}_{n=-\infty}^{\infty}, \quad e_{n}(x)=e^{i n q x} / \sqrt{l}, \quad q=2 \pi / l, \quad n=0, \pm 1, \pm 2, \cdots .
$$

Define a family of projections $P_{N}: L^{2}(0, l) \longrightarrow L^{2}(0, l)(N=0,1, \cdots)$ by

$$
P_{N} \phi=\phi_{N}=\sum_{n=-N}^{N} c_{n} e_{n}, \quad \text { where } \phi=\sum_{n=-\infty}^{\infty} c_{n} e_{n} \in L^{2}(0, l)
$$


The semi-discrete Galerkin approximation to Eq.(2.1) is:

$$
\left\{\begin{array}{c}
\partial_{t} \psi_{N}=\left(i+c_{0}\right) D^{2} \psi_{N}+\left(c_{0} / c_{1}\right) \psi_{N} \\
+\left(i-c_{0} / c_{1}\right) P_{N}\left(\left|\psi_{N}\right|^{2} \psi_{N}\right),-\infty<x<\infty, t>0, \\
\psi_{N}(x, 0)=\psi_{N}^{0}(x), \quad-\infty<x<\infty,
\end{array}\right.
$$

where, and in the sequel, we use the notation $\partial_{t}=\partial / \partial t, D^{k}=\partial^{k} / \partial x, k=0,1,2, \cdots$.

On setting

$$
\begin{gathered}
\psi_{N}(x, t)=\sum_{n=-N}^{N} a_{N, n}(t) e_{n}(x), \\
\psi_{N}^{0}(x)=\sum_{n=-N}^{N} a_{n} e_{n}(x),
\end{gathered}
$$

we may rewrite (2.2) in the following more explicit form:

$$
\left\{\begin{aligned}
d a_{N, n} / d t & =-\left(i+c_{0}\right)(n q)^{2} a_{N, n}+\left(c_{0} / c_{1}\right) a_{N, n} \\
& +\left(i-c_{0} / c_{1}\right) \sum_{m_{1}-m_{2}+m_{3}=n} a_{N, m_{1}} a_{N, m_{2}}^{*} a_{N, m_{3}}, t>0, \\
a_{N, n}(0) & =a_{n}, \quad n=0, \pm 1, \cdots, \pm N .
\end{aligned}\right.
$$

This is an initial value problem of a system of ordinary differential equations, and hence, it can be solved numerically with ease by various finite difference methods.

The sequence $\left\{\psi_{N}(x, t)\right\}_{N=0}^{\infty}$ determined by the above Galerkin scheme (2.2) or (2.3) and (2.4) is expected to converge to the solution of (2.1).

In order to proceed further, let us introduce for convenience the complex-valued function spaces for $k \in \mathbf{N}$ :

$$
C_{p}^{k}[0, l]=\left\{U \in C^{k}[0, l] \mid U^{(j)}(0)=U^{(j)}(l), j=0, \cdots, k\right\} \subset C^{k}[0, l]
$$

and

$$
H_{p}^{k}(0, l)=\text { the completion of } C_{p}^{k}[0, \eta] \text { in } W^{k, 2}(0, l) .
$$

The norms of $C_{p}^{k}[0, l]$ and $H_{p}^{k}(0, l)$ will be denoted by ||$_{k}$ and \|\|$_{k}$ respectively.

In [18] we have shown that if $\psi^{0} \in H_{p}^{4}(0, l)$ and

(i) $c_{0}>0, c_{1}>0$, or

(ii) $c_{0}>0, c_{1}<0$, and

$$
\left\|\psi^{0}\right\|_{0}^{2} \leq\left(\sqrt{c_{1}^{2}-4 l^{2} c_{1}}+c_{1}\right) / 2 l
$$

then (2.1) has a unique classical solution $\psi(x, t)$ on $(-\infty, \infty) \times[0, \infty)$ with period $l$ in the spatial variable and satisfying

$$
\sup _{t \geq 0}\|\psi(t)\|_{k} \leq M_{k}, \quad k=0,1
$$

where $M_{k}>0$ is a constant depending only on $c_{0}, c_{1}, l$, and $\left\|\psi^{0}\right\|_{k}$.

The following statement establishes the convergence of the Galerkin approximation. 
THEOREM 2.1. Suppose $\psi^{0} \in H_{p}^{k}(0, l), k \geq 4$ and $\psi(x, t)$ is the unique classical solution of Eq.(2.1). Then, under the condition (i) or (ii), there are constants $\delta, \epsilon>0$ depending only on $c_{0}, c_{1}, k, l$, and $\left\|\psi^{0}\right\|_{k}$ such that for any $N>0$ and $T>0$ we have the error estimates

$$
\sup _{0 \leq t \leq T}\left\|\psi(t)-\psi_{N}(t)\right\|_{j} \leq \delta e^{\epsilon T} / N^{k-j}, \quad j=0, \cdots, k,
$$

where $\left\{\psi_{N}(x, t)\right\}_{N=0}^{\infty}$ is determined through the scheme (2.2) or (2.3) and (2.4).

REMARK 2.2. The error estimates (2.6) of the Galerkin approximation scheme for computing the spatially-periodic solutions of the Ginzburg-Landau equation is optimal in the sense that the error of the numerical solution is of the same order as the error in the initial approximation. In fact, for any function $U \in H_{p}^{k}(0, l)$ and $0 \leq j \leq k$, we have the standard inequality

$$
\left\|U-P_{N} U\right\|_{j} \leq C\|U\|_{k} / N^{k-j},
$$

where $C$ is a constant independent of $N$ and $U$. Note also that our error control here for Galerkin's method is better than that for the pseudospectral approximation [19].

In the remaining part of this section, we show that the initial value problem (2.4) is globally and uniquely solvable for any $N$. Thus, the Galerkin approximation sequence $\left\{\psi_{N}(x, t)\right\}_{N=0}^{\infty}$ is well-defined through (2.3). In the next section we obtain some preliminary a priori estimates for the higher order derivatives of the sequence $\left\{\psi_{N}(x, t)\right\}_{N=0}^{\infty}$ as well as $\psi(x, t)$-the classical solution of the initial value problem (2.1). The inequality (2.6) will be proved in Section 4.

In order to establish the global existence of the solution to (2.4) for any fixed $N$ under the condition of Theorem 2.1, it suffices to prove:

LEMMA 2.3. If the condition (i) or (ii) holds, then, for any fixed $N \geq 0$,

$$
I_{N, 0}(t) \equiv \int_{0}^{l}\left|\psi_{N}(x, t)\right|^{2} d x=\sum_{n=-N}^{N}\left|a_{N, n}(t)\right|^{2} \leq M_{0},
$$

where $M_{0}>0$ is a constant depending only on $c_{0}, c_{1}, l$, and $\left\|\psi^{0}\right\|_{0}$.

Proof. (2.3) and (2.4) or (2.2) may be rewritten in the following more useful form:

$$
\left\{\begin{aligned}
\left(\partial_{t} \psi_{N}, \xi_{N}\right)_{2} & =\left(i+c_{0}\right)\left(D^{2} \psi_{N}, \xi_{N}\right)_{2} \\
& +\left(c_{0} / c_{1}\right)\left(\psi_{N}, \xi_{N}\right)_{2}+\left(i-c_{0} / c_{1}\right)\left(\left|\psi_{N}\right|^{2} \psi_{N}, \xi_{N}\right)_{2} \\
\psi_{N}(x, 0)= & \psi_{N}^{0}(x), \quad N=0,1,2, \cdots
\end{aligned}\right.
$$


where $\xi_{N}(x, t)=\sum_{-N}^{N} b_{n}(t) e_{n}(x)$ is arbitrary.

First, suppose (i) is verified.

Setting $\xi_{N}=\psi_{N}$ in (2.9) we have

$$
d I_{N, 0} / d t=-2 c_{0} \int_{0}^{l}\left|D \psi_{N}\right|^{2} d x+\left(2 c_{0} / c_{1}\right) I_{N, 0}-\left(2 c_{0} / c_{1}\right) \int_{0}^{l}\left|\psi_{N}\right|^{4} d x .
$$

It then yields from (2.10) and the Schwarz inequality that

$$
d I_{N, 0} / d t \leq\left(2 c_{0} / c_{1}\right) I_{N, 0}\left(1-I_{N, 0} / l\right) .
$$

Consequently, one finds the bound

$$
I_{N, 0}(t) \leq \max \left\{I_{N, 0}(0), l\right\} \leq \max \left\{\left\|\psi^{0}\right\|_{0}^{2}, l\right\} .
$$

Now assume (ii) is satisfied.

For fixed $t$, choose $x_{t} \in[0, l]$ such that $\left|\psi_{N}\left(x_{t}, t\right)\right|^{2}=I_{N, 0}(t) / l$. Then

$$
\begin{gathered}
\left|\psi_{N}(x, t)\right|^{2} \leq\left|\psi_{N}\left(x_{t}, t\right)\right|^{2}+2 \int_{0}^{l}\left|\psi_{N} D \psi_{N}\right| d x \\
\leq I_{N, 0} / l+2 I_{N, 0}^{\frac{1}{2}}\left(\int_{0}^{l}\left|D \psi_{N}\right|^{2} d x\right)^{\frac{1}{2}} .
\end{gathered}
$$

Therefore, it follows from a simple interpolation inequality that

$$
\int_{0}^{l}\left|\psi_{N}\right|^{4} d x \leq I_{N, 0}^{2} / l+a I_{N, 0}^{3}+a^{-1} \int_{0}^{l}\left|D \psi_{N}\right|^{2} d x, \forall a>0 .
$$

Substituting (2.12) into (2.10) and taking $a=1 / c_{2}$ with $c_{2}=-c_{1}$ we obtain

$$
d I_{N, 0} / d t \leq\left(2 c_{0} / c_{2}\right)\left(I_{N, 0} / l+I_{N, 0}^{2} / c_{2}-1\right) I_{N, 0} .
$$

One then finds from solving the above differential inequality the bound

$$
I_{N, 0}(t) \leq\left(\sqrt{c_{2}^{2}+4 l^{2} c_{2}}-c_{2}\right) / 2 l, \quad t \geq 0 .
$$

Lemma 2.3 is proved.

3. Estimates for higher order derivatives. Let $\left\{\psi_{N}(x, t)\right\}_{N=0}^{\infty}$ be the approximation sequence determined by the Galerkin scheme (2.2) or (2.3) and (2.4). Denote by $\psi(x, t)$ the unique global classical solution of the spatially-periodic initial value problem (2.1). 
LEMMA 3.1. Suppose that the condition (i) or (ii) is verified. If $\psi^{0} \in H_{p}^{k}(0, l)$, then there is a constant $M_{k}>0$ depending only on $c_{0}, c_{1}, l$, and $\left\|\psi^{0}\right\|_{k}$ such that

$$
\left\|\psi_{N}(t)\right\|_{k} \leq M_{k}, \quad N=0,1,2, \cdots, \quad t>0 .
$$

Proof. (3.1) has been established in Lemma 2.3 for $k=0$.

To simplify the notation, define

$$
I_{N, k}(t)=\left\|D^{k} \psi_{N}(t)\right\|_{0}^{2}, \quad k, N=0,1,2, \cdots, \quad t \geq 0 .
$$

For $k=1$, we have

$$
d I_{N, 1}(t) / d t=-2 \operatorname{Re}\left(\partial_{t} \psi_{N}, D^{2} \psi_{N}\right)_{2} .
$$

Setting $\xi_{N}(x, t)=D^{2} \psi_{N}(x, t)$ in (2.9) and applying the resulting relation in (3.2) we obtain

$$
d I_{N, 1} / d t=-2 c_{0} I_{N, 2}+\left(2 c_{0} / c_{1}\right) I_{N, 1}-2 \operatorname{Re}\left\{\left(i-c_{0} / c_{1}\right)\left(\left|\psi_{N}\right|^{2} \psi_{N}, D^{2} \psi_{N}\right)_{2}\right\}
$$

From (2.11) it follows that

$$
\leq-2 c_{0} I_{N, 2}+\left(2 c_{0} /\left|c_{1}\right|\right) I_{N, 1}+\left(1+c_{0} /\left|c_{1}\right|\right)\left(b \int_{0}^{l}\left|\psi_{N}\right|^{6} d x+b^{-1} I_{N, 2}\right), \quad \forall b>0 .
$$

and thus, using (2.11) and (3.4),

$$
\int_{0}^{l}\left|\psi_{N}\right|^{4} d x \leq I_{N, 0}^{2} / l+2 I_{N, 0}^{\frac{3}{2}} I_{N, 1}^{\frac{1}{2}}
$$

$$
\begin{gathered}
\int_{0}^{l}\left|\psi_{N}\right|^{6} d x \leq\left(\int_{0}^{l}\left|\psi_{N}\right|^{4} d x\right)\left(I_{N, 0} / l+2 I_{N, 0}^{\frac{1}{2}} I_{N, 1}^{\frac{1}{2}}\right) \\
\leq C_{1}+C_{2} I_{N, 1},
\end{gathered}
$$

where $C_{1}, C_{2}>0$ are constants depending only on $M_{0}$ (Lemma 2.3).

In virtue of (2.10), (2.12), (3.3), and (3.5), we can choose appropriate constants $a, b, \mu, \sigma, \gamma>0$ to get, for any $N$, the inequality

$$
d\left(\mu I_{N, 0}+I_{N, 1}\right) / d t \leq-\sigma\left(\mu I_{N, 0}+I_{N, 1}\right)+\gamma, \quad t \geq 0 .
$$

It then follows from (3.6) that

$$
\mu I_{N, 0}(t)+I_{N, 1}(t) \leq\left(\mu I_{N, 0}(0)+I_{N, 1}(0)\right) e^{-\sigma t}+\gamma\left(1-e^{-\sigma t}\right) / \sigma
$$




$$
\leq(1+\mu)\left\|\psi^{0}\right\|_{1}^{2} e^{-\sigma t}+\gamma\left(1-e^{-\sigma t}\right) / \sigma, \quad t \geq 0
$$

Hence (3.1) is verified for the case $k=1$. In particular, using the natural embedding

$$
H_{p}^{k}(0, l) \longrightarrow C_{p}^{k-1}[0, l]
$$

we can find a constant $C_{0}>0$ depending only on $c_{0}, c_{1}, l$, and $\left\|\psi^{0}\right\|_{1}$ such that

$$
\sup _{N, x, t}\left|\psi_{N}(x, t)\right| \leq C_{0}
$$

Suppose Lemma 3.1 is true for $k \leq m$ where $m \geq 1$ is an integer. Assume now $\psi^{0} \in H_{p}^{m+1}(0, l)$. Then

$$
d I_{N, m+1} / d t=2(-1)^{m+1} \operatorname{Re}\left(\partial_{t} \psi_{N}, D^{2(m+1)} \psi_{N}\right)_{2}
$$

In (2.9), set $\xi_{N}=D^{2(m+1)} \psi_{N}$. As a consequence,

$$
\begin{gathered}
\left(\partial_{t} \psi_{N}, D^{2(m+1)} \psi_{N}\right)_{2}=\left(i+c_{0}\right)\left(D^{2} \psi_{N}, D^{2(m+1)} \psi_{N}\right)_{2} \\
+\left(c_{0} / c_{1}\right)\left(\psi_{N}, D^{2(m+1)} \psi_{N}\right)_{2}+\left(i-c_{0} / c_{1}\right)\left(\left|\psi_{N}\right|^{2} \psi_{N}, D^{2(m+1)} \psi_{N}\right)_{2} \\
=(-1)^{m}\left(i+c_{0}\right) I_{N, m+2}+(-1)^{m+1}\left(c_{0} / c_{1}\right) I_{N, m+1} \\
+\left(i-c_{0} / c_{1}\right)\left(\left|\psi_{N}\right|^{2} \psi_{N}, D^{2(m+1)} \psi_{N}\right)_{2} .
\end{gathered}
$$

From (3.8) and Leibniz' rule, it follows easily that

$$
\left|\left(\left|\psi_{N}\right|^{2} \psi_{N}, D^{2(m+1)} \psi_{N}\right)_{2}\right|=\left|\left(D^{m+1}\left(\left|\psi_{N}\right|^{2} \psi_{N}\right), D^{m+1} \psi_{N}\right)_{2}\right| \leq C_{1}+C_{2} I_{N, m+1}
$$

where $C_{1}, C_{2}>0$ are independent of $N$ and $t \geq 0$.

Substituting (3.10) into (3.9) and using (3.11) we obtain the inequality

$$
d\left(\mu I_{N, m}+I_{N, m+1}\right) / d t \leq-\sigma\left(\mu I_{N, m}+I_{N, m+1}\right)+\gamma,
$$

where $\mu, \sigma, \gamma>0$ are suitable constants. The above inequality is similar to (3.6), thus, a constant $A_{m+1}>0$ depending only on $c_{0}, c_{1}, l$, and $\left\|\psi^{0}\right\|_{m+1}$ can be found such that

$$
\sup _{N, t} I_{N, m+1}(t) \leq A_{m+1} \text {. }
$$

So (3.1) is also verified for $k=m+1$ and the proof of Lemma 3.1 is complete. 
LEMma 3.2. Suppose $\psi^{0} \in H_{p}^{4}(0, l) \cap H_{p}^{k}(0, l)$ and the condition (i) or (ii) holds. Then there is a constant $M_{k}>0$ depending only on $c_{0}, c_{1}, l$, and $\left\|\psi^{0}\right\|_{k}$ so that

$$
\|\psi(t)\|_{k} \leq M_{k}, \quad t>0 .
$$

Proof. The cases $k=0,1$ are stated in (2.5). The rest of the proof is analogous to that for Lemma 3.1 .

4. Optimal error estimates. In this section, let $k \geq 0$ be an integer. Define a map $\mathcal{L}: H_{p}^{k}(0, l) \longrightarrow H_{p}^{k}(0, l)$ by

$$
\mathcal{L}=\left(i+c_{0}\right) D^{2}
$$

where $\operatorname{Dom}(\mathcal{L})=H_{p}^{k+2}(0, l) \subset H_{p}^{k}(0, l)$. It is straightforward to check that $-\mathcal{L}$ is accretive on $\operatorname{Dom}(\mathcal{L})$, namely,

$$
\operatorname{Re}(-\mathcal{L} \phi, \phi)_{H_{p}^{k}} \geq 0, \quad \phi \in \operatorname{Dom}(\mathcal{L}),
$$

and $\operatorname{Ker}(I-\mathcal{L})=\{0\}$, where the condition $c_{0}>0$ has been used. Therefore, $I-\mathcal{L}$ is surjective and hence $\mathcal{L}$ is a generator of a contraction semigroup $\{S(t) \mid t \geq 0\}$ on $H_{p}^{k}(0, l)$ (cf. [14]).

Define

$$
\mathcal{M} \phi=\left(c_{0} / c_{1}\right) \phi+\left(i-c_{0} / c_{1}\right)|\phi|^{2} \phi .
$$

We can now rewrite (2.1) and (2.2) as the Bochner integral equations

$$
\psi(t)=S(t) \psi^{0}+\int_{0}^{t} S(t-s) \mathcal{M} \psi(s) d s
$$

and

$$
\psi_{N}(t)=S(t) P_{N} \psi^{0}+\int_{0}^{t} S(t-s) P_{N} \mathcal{M} \psi_{N}(s) d s
$$

in the space $H_{p}^{k}(0, l)$.

Let $\epsilon_{N}(t)=\psi(t)-\psi_{N}(t)$ be the error of the approximation. Then we have the decomposition

$$
\begin{gathered}
\epsilon_{N}(t)=S(t)\left(I-P_{N}\right) \psi^{0}+\int_{0}^{t} S(t-s)\left(I-P_{N}\right) \mathcal{M} \psi(s) d s \\
+\int_{0}^{t} S(t-s) P_{N}\left(\mathcal{M} \psi(s)-\mathcal{M} \psi_{N}(s)\right) d s \\
\equiv \epsilon_{N}^{1}(t)+\epsilon_{N}^{2}(t)+\epsilon_{N}^{3}(t)
\end{gathered}
$$

From (2.7) we find for $0 \leq j \leq k$,

$$
\left\|\epsilon_{N}^{1}(t)\right\|_{j} \leq C_{1}\left\|\psi^{0}\right\|_{k} / N^{k-j}, \quad \forall t,
$$


and using Leibniz' rule, Lemma 3.2, and the embedding (3.7),

$$
\sup _{0 \leq t \leq T}\left\|\epsilon_{N}^{2}(t)\right\|_{j} \leq C_{2} T / N^{k-j}
$$

where $C_{2}$ depends only on $c_{0}, c_{1}, k, l$, and $\left\|\psi^{0}\right\|_{k}$.

On the other hand,

$$
\mathcal{M} \psi-\mathcal{M} \psi_{N}=\left(c_{0} / c_{1}\right) \epsilon_{N}+\left(i-c_{0} / c_{1}\right)\left(\epsilon_{N}\left(|\psi|^{2}+\left|\psi_{N}\right|^{2}\right)+\epsilon_{N}^{*} \psi \psi_{N}\right)
$$

so, applying Leibniz' rule, Lemmas $3.1,3.2$, and the embedding (3.7), we get

$$
\left\|\epsilon_{N}^{3}(t)\right\|_{j} \leq C_{3} \int_{0}^{t}\left\|\epsilon_{N}(s)\right\|_{j} d s, \quad t>0
$$

where $C_{3}>0$ is a constant depending only on $c_{0}, c_{1}, l,\left\|\psi^{0}\right\|_{1}$, and $\left\|\psi^{0}\right\|_{j}$.

Finally, inserting the bounds (4.2)-(4.4) into (4.1) and using the Gronwall inequality, we obtain immediately an error estimation inequality of the type (2.6). Therefore Theorem 2.1 is proved.

Note that pointwise error estimates for the Galerkin approximation may also be obtained by Theorem 2.1 and the embedding (3.7).

5. Numerical experiments on finite dimensionality of waves. Using the convergent Galerkin scheme (2.2) or (2.3) and (2.4) as a numerical approximation tool, we can observe by computer experiments the finite dimensionality of wave motions of the Ginzburg-Landau equation (in the parameter region $c_{0}, c_{1}>0$ ) established in [3,1].

To simplify the analysis a little bit, let us consider solutions of (2.1) evolving from a small neighbourhood of $\psi=0$. Linearisation of (2.1) around $\psi=0$ leads to the equation:

$$
\eta_{t}=\mathcal{A} \eta \equiv\left(i+c_{0}\right) \eta_{x x}+\left(c_{0} / c_{1}\right) \eta, \quad-\infty<x<\infty, \quad t>0
$$

It is easily calculated that the eigenspectrum of $\mathcal{A}$ is:

$$
\sigma(\mathcal{A})=\left\{-c_{0}\left(n^{2} q^{2}-1 / c_{1}\right)-i n^{2} q^{2} \mid n=0,1,2, \cdots\right\},
$$

with the corresponding set of eigenfunctions $\left\{e^{ \pm i n q x} \mid n=0,1,2, \cdots\right\}$. Hence, according to the standard linear stability analysis, high frequency spatial modes with $n^{2} q^{2}>1 / c_{1}$ in the solution

$$
\psi(x, t)=\sum_{n=-\infty}^{\infty} a_{n}(t) e^{i n q x}
$$

initiated from a neighbourhood of $\psi=0$ will be damped. Consequently, if the linear theory can give a qualitatively correct description of the solutions of $(2.1)$ when $\psi^{0}$ is 
small, we may predict that high modes with $n^{2} q^{2}>1 / c_{1}$ will be absent after a long time development. In the following, we examine the above prediction via computer experiments using the scheme (2.2) or (2.3) and (2.4).

For definiteness, we put $c_{0}=0.2, c_{1}=1.0, \psi^{0}(x)=0.1 \cos q x+0.1 \cos 2 q x$ and vary $q$. In our procedure, we first solve (for various integer $N$ ) the system (2.4) numerically by the fourth-order Runge-Kutta method. Then the Galerkin approximation sequence $\left\{\psi_{N}\right\}_{N=0}^{\infty}$ is constructed via (2.3). Our numerical results can briefly be illustrated as follows. (To convince the reader, we shall present a set of three-dimensional and level-line graphs, rather than provide a frequency analysis for the spatial modes, of $\psi$. Only the real component of $\psi$ will be exhibited. The imaginary part has the similar behaviour, and hence, is omitted here.)

EXAMPLE 5.1. Set $q=0.4$. Linear theory prediction: Spatial modes with $|n|>2$ do not exist after a long time. In order to have a numerical confirmation we take $T=60$. A computation through Galerkin's scheme on the time interval $[0, T]$ is carried out. The approximation is already good for $N \geq 8$. Figure 1 is obtained by taking $N=16$.

EXAMPLE 5.2. Choose $q=0.8$. Linear theory prediction: The only remaining spatial modes after a long time evolution are those with $|n| \leq 1$. To have a mumerical observation we take $T=60$. Galerkin's approximation is already good for $N \geq 7$. Figure 2 is obtained by taking $N=16$. These graphs show that, after a period of time, simple modes with $|n| \leq 1$ become dominating. Hence a much clearer verification of the above linear theory assertion is reached.

EXAMPLE 5.3. Put $q=1.5$. Linear theory prediction: After a long time development all spatially-dependent modes will be exhausted and the motion will only depend on time. To have a numerical confirmation, we choose $T=60$. The Galerkin approximation is already good for $N \geq 5$. Figure 3 is obtained by fixing $N=16$. These graphs verify perfectly the prediction made from the linear theory. Figure 3 also reveals that the zero solution $\psi=0$ is unstable as can be examined readily by the Lyapunov method.

REMARKS 5.4. (a) These examples show that finite dimensional motions may be observed after a finite time evolution and the linear theory seems to be fairly accurate to provide a good estimate of the number of dimensions of the wave motions.

(b) More computer experiments show that high modes may participate in the flow initiated from a very simple spatial state after a period of time provided $q$ is sufficiently small.

(c) In Examples 5.1 and 5.2, the Stokes wave $\Psi=e^{i t}$ is linearly unstable under spatially-periodic disturbances of the wavelength $l=2 \pi / q$, while, in Example $5.3, \Psi$ is linearly stable. In the range $1<q^{2}<192 / 104$ where $\Psi$ is linearly unstable, one should 
not be surprised to see that the heuristic assertion of Example 5.3 made based upon linear analysis may fail.

6. Zero dissipation limit. In this section, we prove that, under the condition (i) or (ii) (Section 2), the solutions of the Ginzburg-Landau equation (2.1) will approach on any finite time interval the solution of the cubic Schrödinger equation

$$
\left\{\begin{array}{l}
\phi_{t}=i \phi_{x x}+i|\phi|^{2} \phi, \quad-\infty<x<\infty, \quad t>0 \\
\phi(x, 0)=\psi^{0}(x), \quad \psi^{0}(x)=\psi^{0}(x+l), \quad-\infty<x<\infty
\end{array}\right.
$$

in the zero dissipation limit $c_{0} \rightarrow 0^{+}$. In our discussion below, we shall first obtain some $c_{0}$-independent a priori bounds for the solutions of (2.1), and then, we pass to the $c_{0} \rightarrow 0^{+}$ limit.

To emphasize the dependence of the solution of Eq.(2.1) on $c_{0}>0$, we write the solution as $\psi\left(c_{0} ; x, t\right)$. We now construct a family of energy functionals:

$$
I_{k}\left(c_{0} ; t\right)=\int_{0}^{l}\left|D^{k} \psi\left(c_{0} ; x, t\right)\right|^{2} d x, \quad k=0,1,2, \cdots
$$

and

$$
E\left(c_{0} ; t\right)=I_{1}\left(c_{0} ; t\right)-\frac{1}{2} \int_{0}^{l}\left|\psi\left(c_{0} ; x, t\right)\right|^{4} d x
$$

Then,

$$
\begin{gathered}
d E\left(c_{0} ; t\right) / d t=-2 c_{0} I_{2}\left(c_{0} ; t\right)+\left(2 c_{0} / c_{1}\right) I_{1}\left(c_{0} ; t\right)-\left(2 c_{0} / c_{1}\right) \int_{0}^{l}|\psi|^{4} d x \\
+\left(2 c_{0} / c_{1}\right) \int_{0}^{l}|\psi|^{6} d x+c_{0}\left(1 / c_{1}-1\right) \int_{0}^{l}|\psi|^{2}\left(\psi^{*} \psi_{x x}+\psi \psi_{x x}^{*}\right) d x .
\end{gathered}
$$

Using the simple interpolation inequality

$$
\left.\left|\int_{0}^{l}\right| \psi\right|^{2} \psi^{*} \psi_{x x} d x \mid \leq \frac{1}{2}\left(a I_{2}\left(c_{0} ; t\right)+a^{-1} \int_{0}^{l}|\psi|^{6} d x\right)
$$

in (6.2) and setting $a=4 /\left(1+1 /\left|c_{1}\right|\right)$, we obtain

$$
d E\left(c_{0} ; t\right) / d t \leq\left(2 c_{0} /\left|c_{1}\right|\right) I_{1}+\left(2 c_{0} /\left|c_{1}\right|\right) \int_{0}^{l}|\psi|^{4} d x+c_{0}\left(\frac{2}{\left|c_{1}\right|}+\frac{1}{8}\left(1+\frac{1}{\left|c_{1}\right|}\right)^{2}\right) \int_{0}^{l}|\psi|^{6} d x
$$

It was shown in [18] that a constant $C>0$ independent of $c_{0}$ can be found so that

$$
\sup _{t} I_{0}\left(c_{0} ; t\right) \leq C \text {. }
$$


On the other hand, by the method used in deriving (2.12) and (3.5), we easily obtain constants $C_{1}, C_{2}>0$ depending only on $\sup _{t} I_{0}\left(c_{0} ; t\right)$ such that

$$
\max \left\{\int_{0}^{l}|\psi|^{4} d x, \int_{0}^{l}|\psi|^{6} d x\right\} \leq C_{1}+C_{2} I_{1}\left(c_{0} ; t\right) .
$$

Hence, (6.3), (6.4), and (6.5) imply:

$$
d E\left(c_{0} ; t\right) / d t \leq c_{0}\left(C_{1}^{\prime} E\left(c_{0} ; t\right)+C_{2}^{\prime}\right)
$$

where $C_{1}^{\prime}, C_{2}^{\prime}>0$ are independent of $c_{0}$.

In virtue of (6.6) and the inequality

$$
\int_{0}^{l}|\psi|^{4} d x \leq I_{0}^{2} / l+2 I_{0}^{\frac{8}{2}} I_{1}^{\frac{1}{2}}
$$

(cf. (3.4)), we immediately get, for any $T>0$, the control

$$
\sup _{0 \leq t \leq T} I_{1}\left(c_{0} ; t\right) \leq C_{T}
$$

where $C_{T}>0$ is a constant depending on $T$ but independent of $c_{0}$.

Eqs.(2.1) and (6.1) can be rewritten in the forms:

$$
\psi\left(c_{0} ; t\right)=e^{t\left(i+c_{0}\right) D^{2}} \psi^{0}+\int_{0}^{t} e^{(t-s)\left(i+c_{0}\right) D^{2}} \mathcal{M} \psi\left(c_{0} ; s\right) d s
$$

where $\mathcal{M}$ is as defined in Section 4 , and

$$
\phi(t)=e^{t i D^{2}} \psi^{0}+i \int_{0}^{t} e^{(t-s) i D^{2}}|\phi(s)|^{2} \phi(s) d s .
$$

As a consequence, we have the decomposition

$$
\begin{gathered}
\epsilon\left(c_{0} ; t\right) \equiv \psi\left(c_{0} ; t\right)-\phi(t) \\
=\left(e^{t c_{0} D^{2}}-I\right) e^{t i D^{2}} \psi^{0}+\int_{0}^{t} e^{(t-s)\left(i+c_{0}\right) D^{2}}\left(\mathcal{M} \psi\left(c_{0} ; s\right)-\mathcal{M} \phi(s)\right) d s \\
+\int_{0}^{t}\left(e^{(t-s) c_{0} D^{2}}-I\right) e^{(t-s) i D^{2}} \mathcal{M} \phi(s) d s+\int_{0}^{t} e^{(t-s) i D^{2}}\left(\mathcal{M} \phi(s)-i|\phi(s)|^{2} \phi(s)\right) d s \\
\equiv \epsilon^{1}\left(c_{0} ; t\right)+\epsilon^{2}\left(c_{0} ; t\right)+\epsilon^{3}\left(c_{0} ; t\right)+\epsilon^{4}\left(c_{0} ; t\right)
\end{gathered}
$$


It can be verified that

$$
\begin{gathered}
\epsilon_{T}^{1}\left(c_{0}\right) \equiv \sup _{0 \leq t \leq T}\left\|\epsilon^{1}\left(c_{0} ; t\right)\right\|_{1} \longrightarrow 0 \quad \text { as } c_{0} \longrightarrow 0^{+}, \\
\left\|\epsilon^{2}\left(c_{0} ; t\right)\right\|_{1} \leq C_{1} \int_{0}^{t}\left\|\epsilon\left(c_{0} ; s\right)\right\|_{1} d s, \quad 0 \leq t \leq T,
\end{gathered}
$$

where $C_{1}>0$ depends on $\sup _{0 \leq t \leq T}\left\|\psi\left(c_{0} ; t\right)\right\|_{1}$ and $\left\|\psi^{0}\right\|_{1}$ but is independent of $0<c_{0}<1$,

$$
\epsilon_{T}^{3}\left(c_{0}\right) \equiv \sup _{0 \leq t \leq T}\left\|\epsilon^{3}\left(c_{0} ; t\right)\right\|_{1} \longrightarrow 0 \quad \text { as } c_{0} \longrightarrow 0^{+}
$$

and

$$
\sup _{0 \leq t \leq T}\left\|\epsilon^{4}\left(c_{0} ; t\right)\right\|_{1} \leq c_{0} T C_{2},
$$

where $C_{2}>0$ depends only on $\left\|\psi^{0}\right\|_{1}$.

It then follows from applying the above results in (6.8) and using Gronwall's inequality that

$$
\left\|\epsilon\left(c_{0} ; t\right)\right\|_{1} \leq\left(\epsilon_{T}^{1}\left(c_{0}\right)+\epsilon_{T}^{3}\left(c_{0}\right)+c_{0} T C_{2}\right) e^{C_{1} T, \quad 0 \leq t \leq T .}
$$

In summary, we have:

THEOREM 6.1. Let $\psi\left(c_{0} ; x, t\right)$ and $\phi(x, t)$ be the solutions of the spatially-periodic initial value problems of the Ginzburg-Landau equation (2.1) and the cubic Schrödinger equation (6.1), respectively. Then, on any finite time interval $[0, T]$,

$$
\lim _{c_{0} \rightarrow 0+} \sup _{0 \leq t \leq T}\left\|\psi\left(c_{0} ; t\right)-\phi(t)\right\|_{1}=0
$$

As an obvious implication of Theorem 6.1 and the embedding (3.7), we have the following pointwise limit

$$
\lim _{c_{0} \rightarrow 0^{+}-\infty<x<\infty, 0 \leq t \leq T} \sup _{-}\left|\psi\left(c_{0} ; x, t\right)-\phi(x, t)\right|=0
$$

NOTE. It can be seen that the condition (ii) may further be relaxed for the parameter region $c_{0}>0, c_{1}<0$ if we restrict ourselves to certain special classes of solutions. For example, if $\psi^{0}$ is odd, results on the global existence, optimal error estimates of Galerkin's scheme, and zero dissipation limit hold provided $\left\|\psi^{0}\right\|_{0} \leq\left|c_{1}\right|^{\frac{1}{4}}$. Moreover, $\psi\left(c_{0} ; x, t\right) \rightarrow 0$ as $t \rightarrow 0$ uniformly with respect to $x \in(-\infty, \infty)$ in the case $\left\|\psi^{0}\right\|_{0}<\left|c_{1}\right|^{\frac{1}{4}}$.

Acknowledgements. The author is grateful to Joel Spruck for valuable suggestions and to Wiktor Szczyrba for his help in the course of making numerical experiments. He would also like to thank Darryl Holm for communicating [1] and Yuji Kodama for pointing out [13]. 


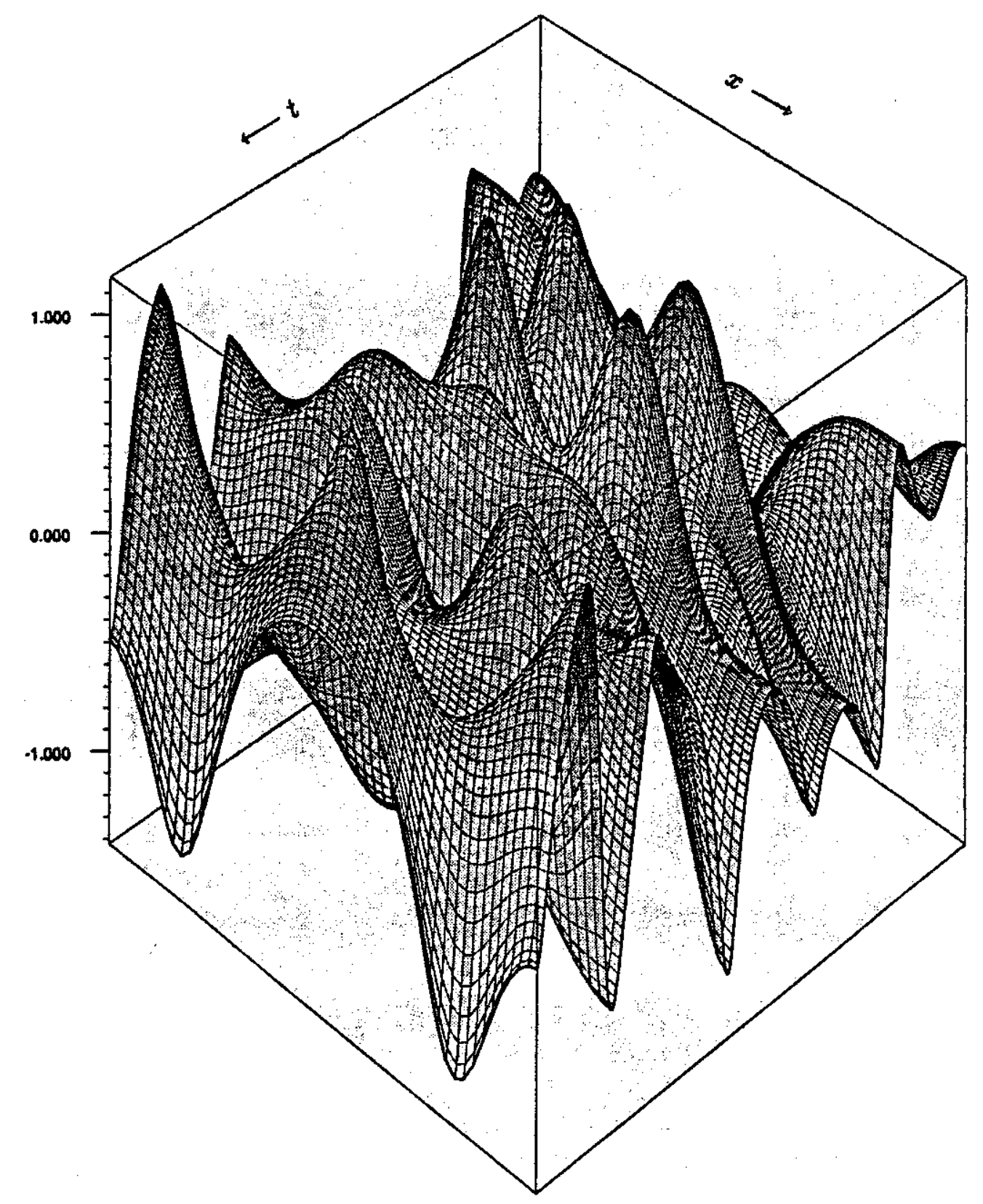

FIGURE 1 (a): A 3D graph of Re $\psi$.

$q=0.4,0 \leq x \leq 2 \pi / q, 0 \leq t \leq 60$. 


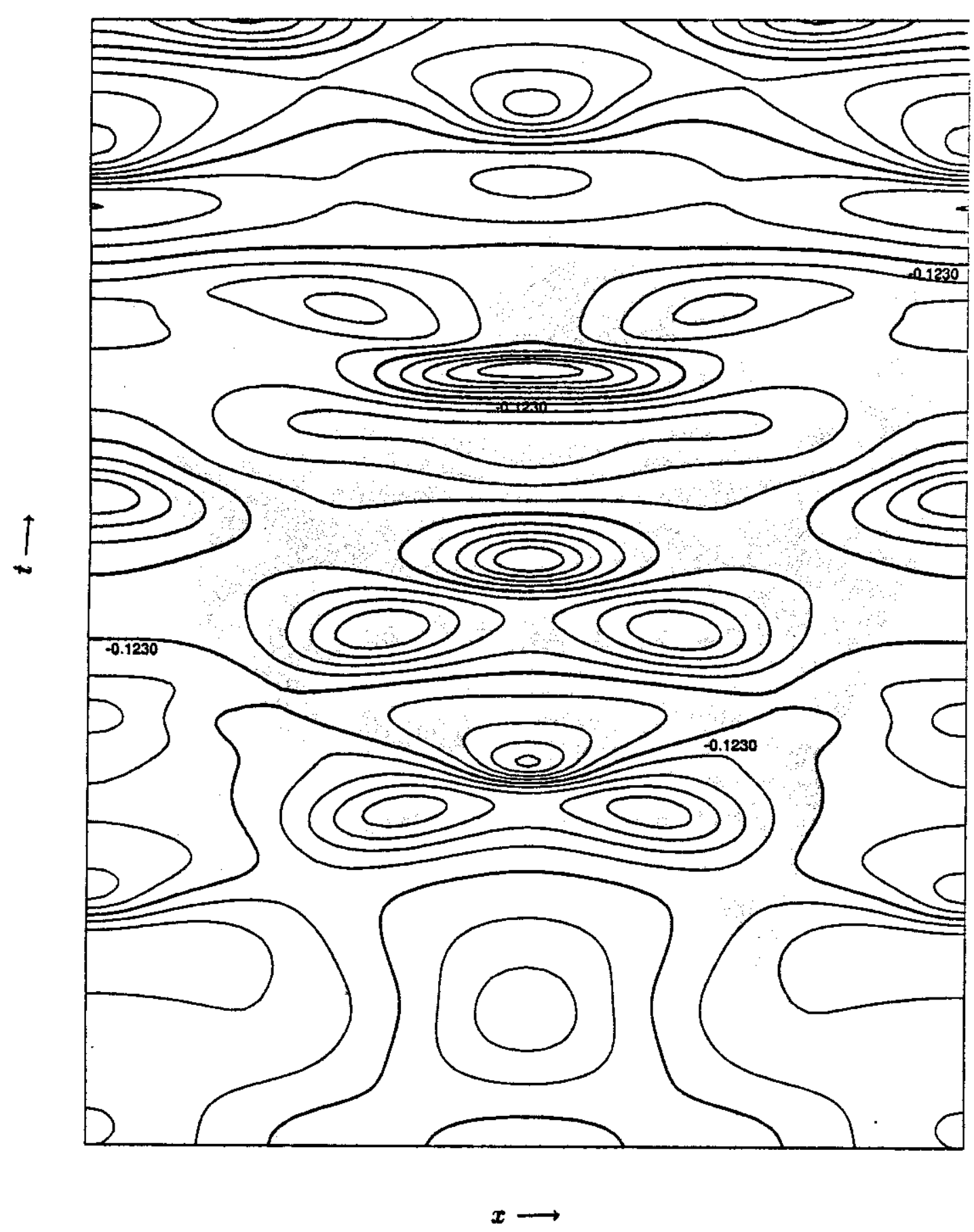

Figure 1 (b): A level-line graph of $\operatorname{Re} \psi$.

$$
q=0.4,0 \leq x \leq 2 \pi / q, 0 \leq t \leq 60 .
$$




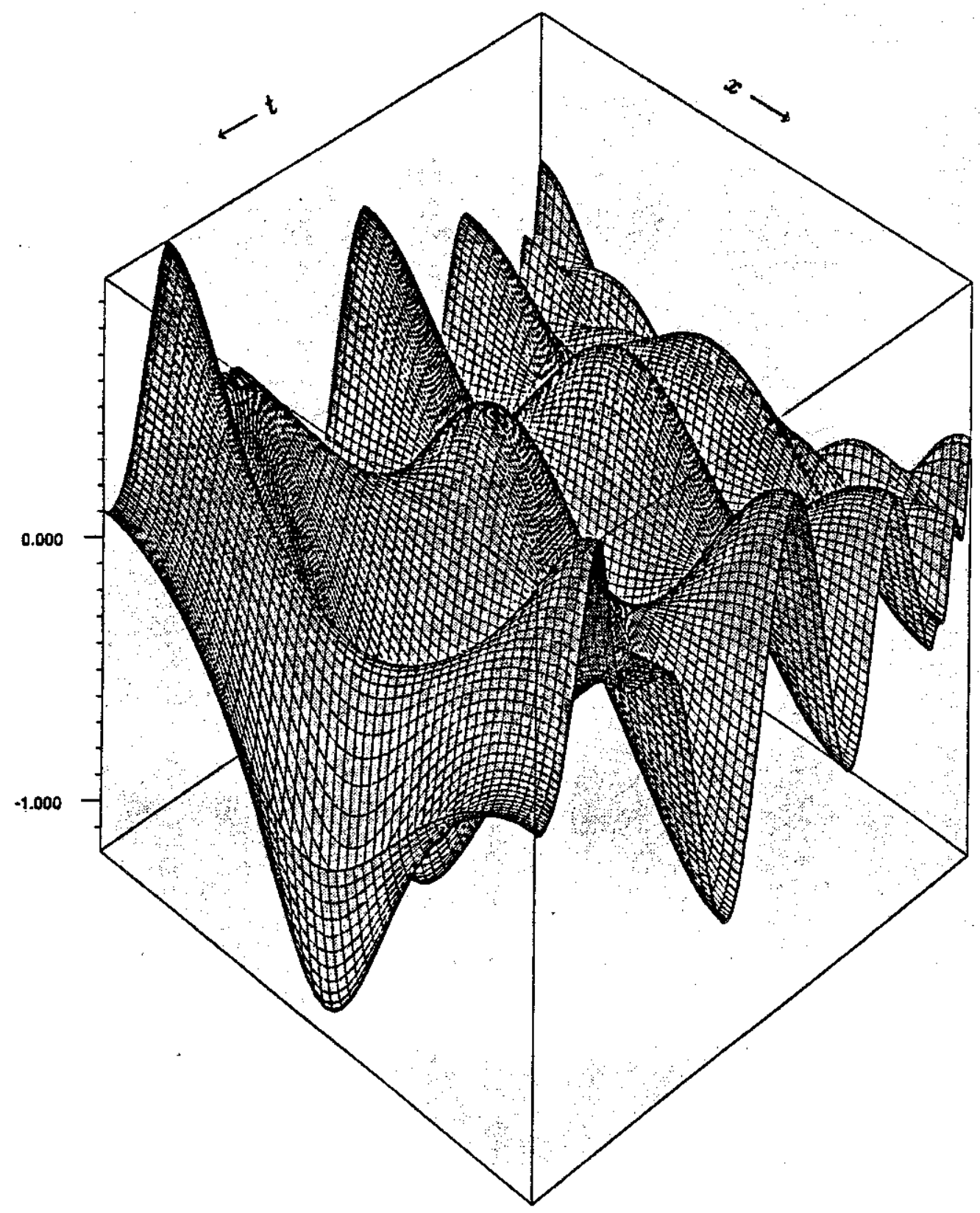

Figure 2 (a): A 3D graph of $\operatorname{Re} \psi$.

$q=0.8,0 \leq x \leq 2 \pi / q, 0 \leq t \leq 60$. 


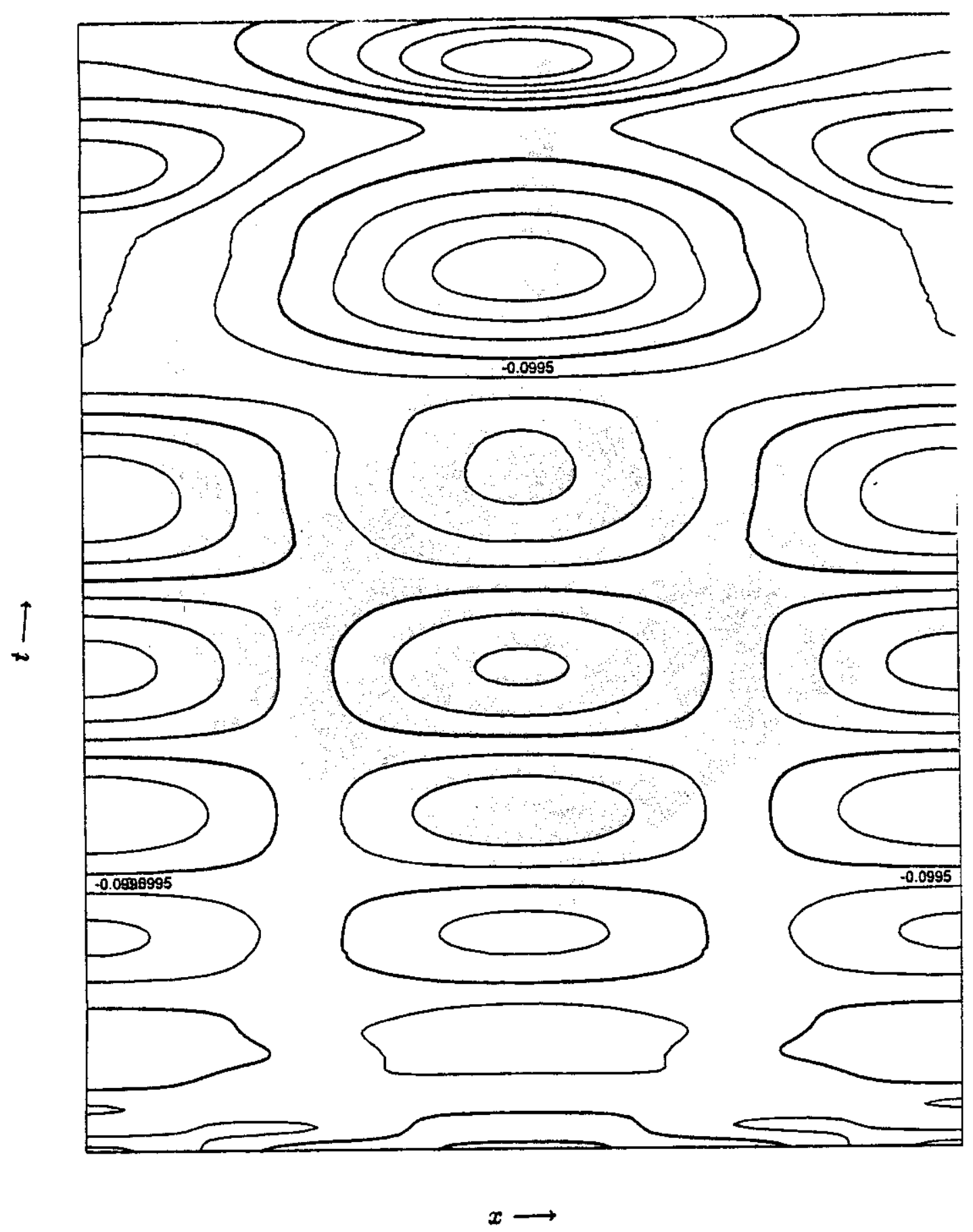

Figure 2 (b): A level-line graph of $\operatorname{Re} \psi$.

$$
q=0.8,0 \leq x \leq 2 \pi / q, 0 \leq t \leq 60 .
$$




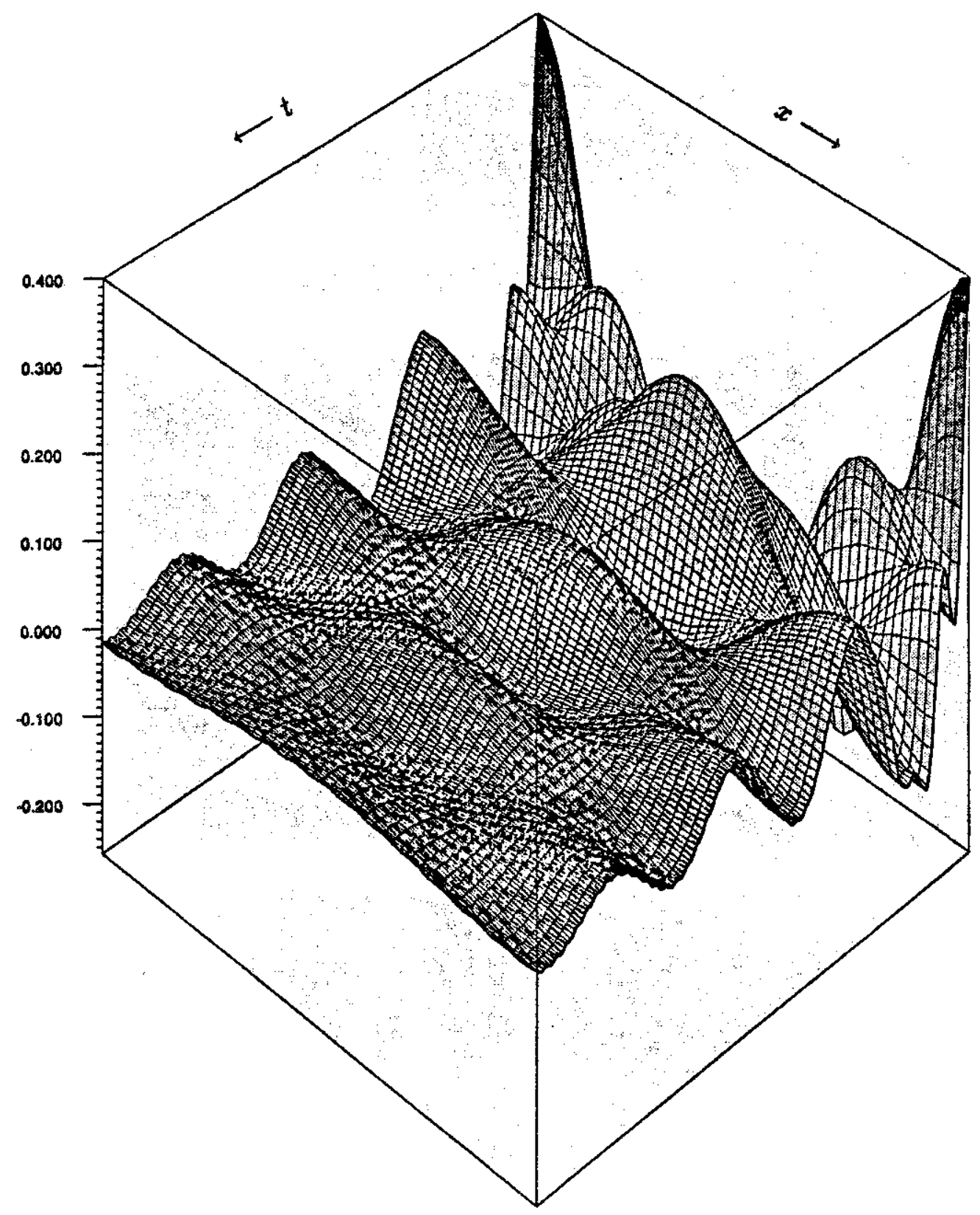

Figure 3 (a): A 3D graph of $\operatorname{Re} \psi$.

$q=1.5,0 \leq x \leq 2 \pi / q, 0 \leq t \leq 10$. 


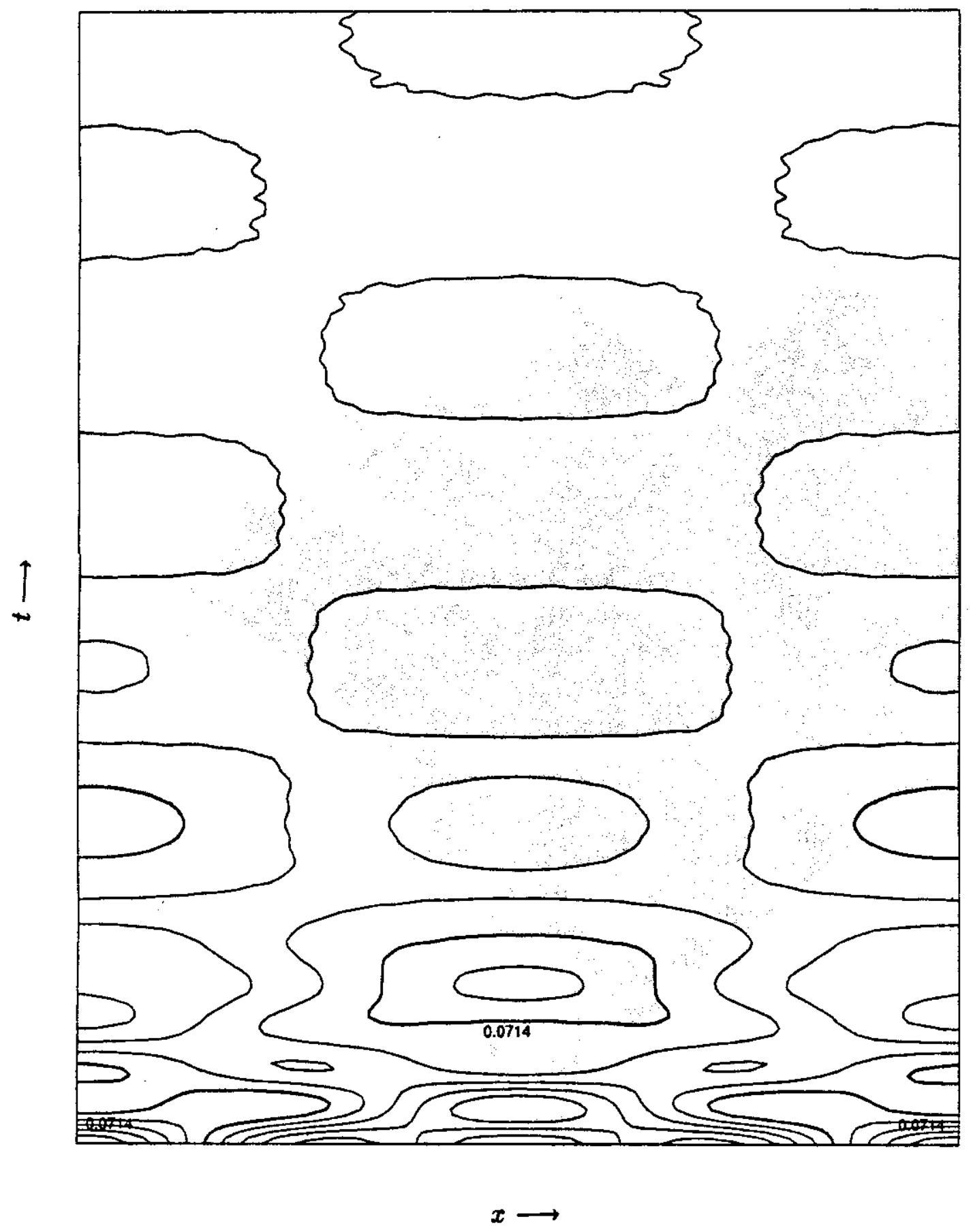

Figure 3 (b): A level-line graph of Re $\psi$. $q=1.5,0 \leq x \leq 2 \pi / q, 0 \leq t \leq 10$. 


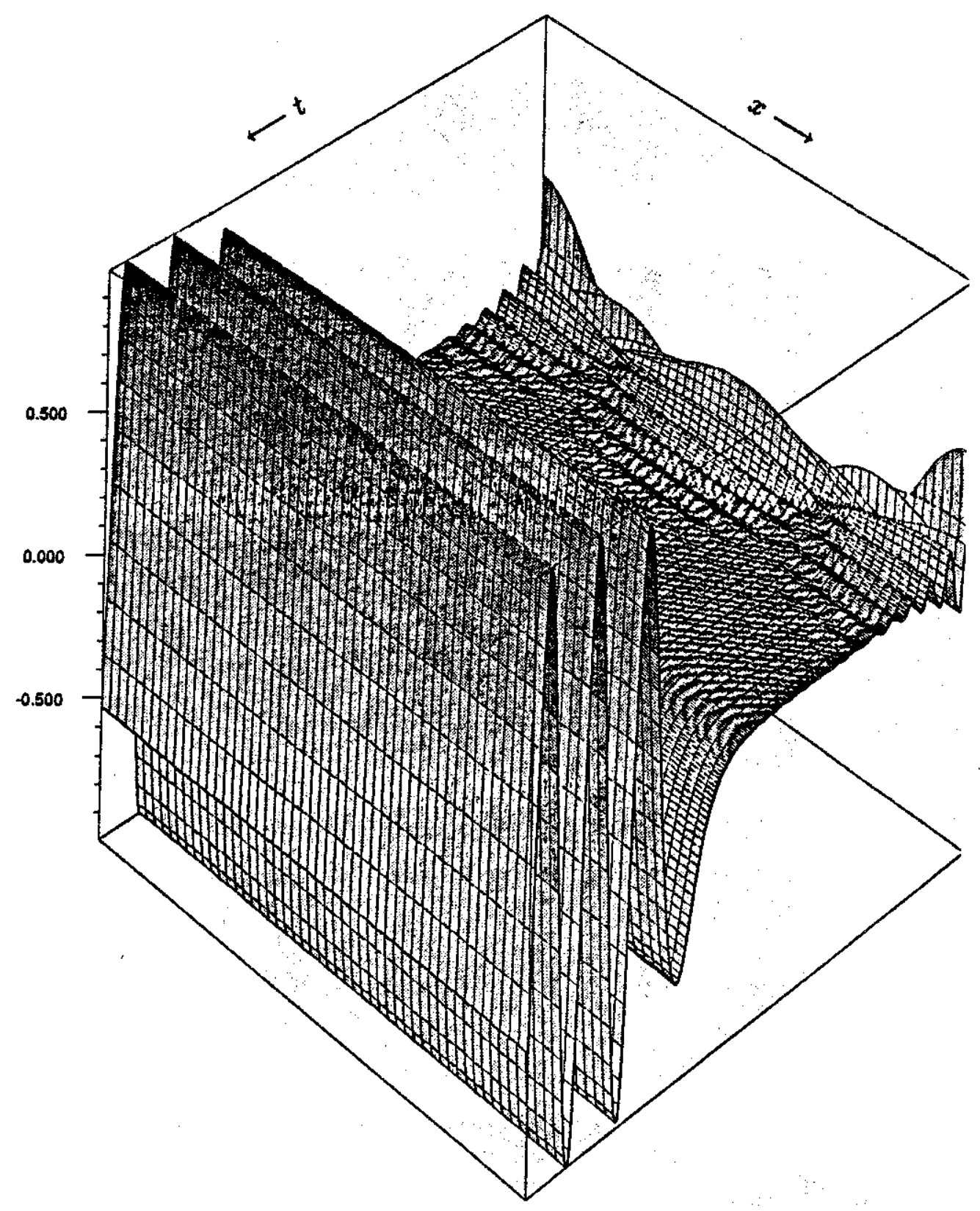

Figure 3 (c): A 3D graph of Re $\psi$.

$q=1.5,0 \leq x \leq 2 \pi / q, 0 \leq t \leq 60$. 
Find

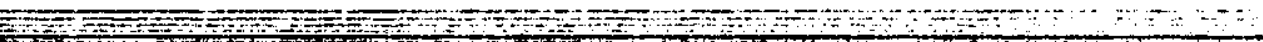

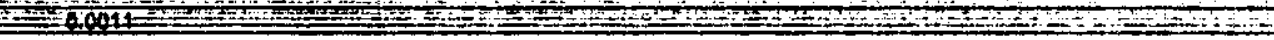

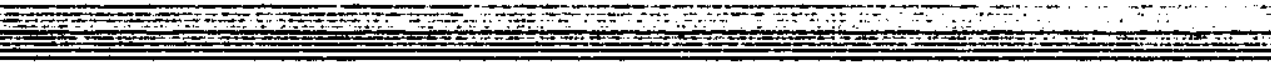
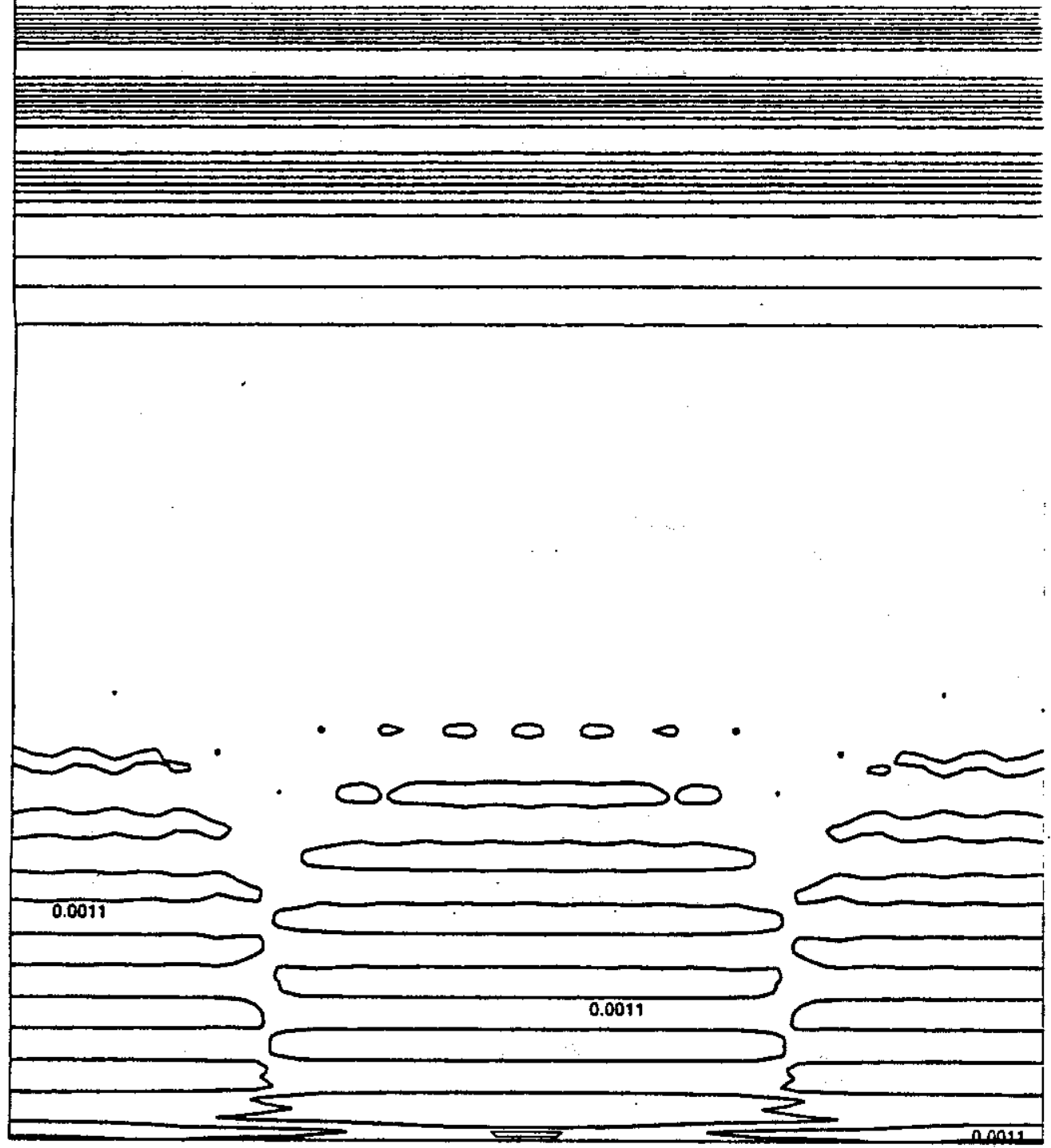

$x \longrightarrow$

Figure 3 (d): A level-line graph of $\operatorname{Re} \psi$. $q=1.5,0 \leq x \leq 2 \pi / q, 0 \leq t \leq 60$. 


\section{REFERENCES}

[1] C. R. Doering, J. D. Gibbon, D. D. Holm, B. Nicolaenko, Low-dimensional behaviour in the complex Ginzburg-Landau equation, Nonlinearity 1 (1988), 279-301.

[2] B. Fornberg, G. B. Whitham, A numerical and theoretical study of certain nonlinear wave phenomena, Philos. Trans. Roy. Soc. London Ser. A 289 (1978), 373-404.

[3] J. M. GHIDAGLIA, B. HÊRoN, Dimension of the attractors associated to the Ginzburg-Landau partial differential equation, Physica D 28 (1987), 282-304.

[4] L. R. KeEfe, Dynamics of perturbed wavetrain solutions to the Ginzburg-Landau equation, Stud. Appl. Math. 73(1985), 91-153.

[5] H. -O. KREISS, J. OuIGER, Comparison of accurate methods for the integration of hyperbolic equations, Tellus 24 (1972), 119-215.

[6] S. Kogelman; R. C. DiPrima; Stability of spatially periodic supercritical flows in hydrodynamics, Phys. Fluids 13 (1970), 1-11.

[7] Y. Kuramoto, T. Yamada, Turbulent state in chemical reactions, Prog. Theoret. Phys. 56 (1976), 679-681.

[8] P. D. LaX, C. D. Levermore, The small dispersion limit of the Korteweg-de Vries equation, I, II, III, Comm. Pure. Appl. Math. 36 (1983), 253-290, 571-593, 809-829.

[9] H. T. Moon, P. Huerre, L. G. REDEKopP, Three-frequency motion and chaos in the Ginzburg-Landau equation, Phys. Rev. Lett. 49 (1982), 458-460.

[10] H. T. Moon, P. Huerre, L. G. Redekopp, Transition to chaos in the Ginzburg-Landau equation, Physica D 7 (1983), 135-150.

[11] A. C. Newell, J. A. Whitehead, Finite bandwidth, finite amplitude convection, J. Fluid Mech. 38 (1969), 279-303.

[12] P. K. Newton, L. Strovich, Instabilities of the Ginzburg-Landau equation: periodic solutions, Quart. Appl. Math. 44 (1986), 49-58.

[13] K. NozaKI, N. BEKKI, Exact solutions of the generalized Ginzburg-Landau equation, J. Phys. Soc. Janpan 53 (1984), 1581-1582.

[14] R. E. Showalter, Hilbert Space Methods for Partial Differential Equations, San Francisco, Pitman, 1979.

[15] L. Sirovich, P. K. Newton, Periodic solutions of the Ginzburg-Landau equation, Physica D 21 (1986), 115-125.

[16] K. Stewartson, J. T. Stuart, A nonlinear instability theory for a wave system in plane Poiseuille flow, J. Fluid Mech. 18 (1971), 529-545.

[17] S. VENAKIDES, The zero dispersion limit of the Korteweg-de Vries equation with periodic initial data, Trans. Amer. Math. Soc. 301 (1987), 189-226.

[18] Y. YANG, Global spatially periodic solutions to the Ginzburg-Landau equation, Proc. Roy. Soc. Edinburgh 110A (1988), 263-273.

[19] Y. YANG, Convergence of the pseudospectral method for the Ginzburg-Landau equation, J. Math. Anal. Appl. (to appear).

[20] V. E. ZAKHARov, A. B. SHABAT, Exact theory of two-dimensional self-focusing and one-dimensional self-modulation of waves in nonlinear media, Soviet Phys. JEPT 34 (1972), 62-69. 